\title{
Numerical Analysis of Dielectric Optical Waveguides
}

\author{
João Paulo N. Torres, Carlos A. F. Fernandes and Ricardo A. Marques Lameirinhas
}

\section{ABSTRACT}

The stochastic resonance (SR) in direct-modulated laser diodes is investigated using an analytical approach based on the laser rate equations for the carrier and photon densities, that include the cross correlation between the photon and carrier noises. This subject may be particularly important in the domain of Optical Communication Systems, where increasing demands on laser performance have led to the fabrication of more and more complex structures. In viewing the development of the associated technology, the importance of the simulation tools revealed of crucial importance.

Keywords: Lasers, Optical Communications, Optics, Optoelectronic Devices, Ring Waveguides.

Published Online: January 4, 2021

ISSN: $2684-4451$

DOI :10.24018/ejphysics.2021.3.1.43

João Paulo N. Torres

Department of Electrical and Computer Engineering, Instituto Superior Técnico, Lisbon, Portugal.

Instituto de Telecomunicações, Lisbon, Portugal.

(e-mail: joaotorres@tecnico.ulisboa.pt)

Carlos A. F. Fernandes

Department of Electrical and Computer Engineering, Instituto Superior Técnico, Lisbon, Portugal.

Instituto de Telecomunicações, Lisbon, Portugal.

(e-mail: ffernandes@tecnico.ulisboa.pt)

Ricardo A. Marques Lameirinhas*

Department of Electrical and Computer Engineering, Instituto Superior Técnico, Lisbon, Portugal.

Instituto de Telecomunicações, Lisbon, Portugal.

(e-mail:

ricardo.lameirinhas @ tecnico.ulisboa.pt)

*Corresponding Author

\section{MODEL}

\section{INTRODUCTION}

Stochastic Resonance (SR) is a topic of increasing interest in several scientific domains [1]-[4], being intensively investigated since it has been referred for the first time in 1981 [1], [2]. Recent works have shown that this subject is also relevant in linear systems [5]-[6], suggesting that the effect of SR on signal-to-noise ratio (SNR) calculations can be included in laser simulation models [7], for the analysis of the electric signal current modulation and the electro-optical signal conversion inherent to the laser emission process. This may be particularly important in the area of Optical Communication Systems (OCS), where one of the main goals is to insure the single longitudinal mode (SLM) operation, by an adequate design of the laser structure [8]-[15].

In section II of the present paper, we extend the analysis described in [7] to non-rectangular waveguides, by considering a more realistic situation that takes into account a non-linear optical gain versus carrier concentration dependence for the semiconductor description [8]-[15]. In section III, some results concerning the power spectrum and the SNR of the photon density are presented and discussed, considering the device geometry. Finally, in section IV, some conclusions and future work are summarized.
The starting point corresponds to the single-mode rate equations for semiconductor lasers that include the Langevin noise sources. They are given by:

$$
\begin{aligned}
& \frac{d N}{d t}=\frac{J}{q d}-\frac{N}{\tau_{s p}}-\frac{c}{n} g(n) S+F_{n} \\
& \frac{d S}{d t}=\left[\frac{c}{n} \Gamma g(n)-\frac{N}{\tau_{p h}}\right] S+\Gamma \beta \frac{N}{\tau_{s p}}+F_{S}
\end{aligned}
$$

where $J$ is the current density, $N$ is the carrier density, $S$ the photon density, $q$ is the modulus of the electron charge, $d$ is the active layer thickness, $\tau_{s p}$ is the spontaneous-emission lifetime of carriers, $\tau_{p h}$ is photon lifetime, $c$ is the light velocity in vacuum, $F_{n}$ and $F_{s}$ are the carrier and photon noises, respectively, $\beta_{s p}$ is the spontaneous-emission factor, $\Gamma$ is the optical confinement factor and $g$ is the optical gain. The photon lifetime depends on the total optical loss, which includes the internal losses and the mirror losses; the spontaneous emission factor is a measure of the fraction of the spontaneous emission that is coupled to the lasing mode, which is typically in the range $10^{-3}$ to $10^{-4}$. The Langevin 
noise terms are related to the carrier and photon fluctuations in the population densities along the laser cavity. In [7], the optical gain is assumed linearly dependent on the carrier density, according to:

$g(N)=a\left(N-N_{0}\right)$

where a is the gain coefficient and $N_{0}$ is the carrier density at transparency.

In [8], the reflective index $\mathrm{n}$ is considered as a function of the carrier density $\mathrm{N}$, the frequency $\mathrm{f}$ and the temperature $\mathrm{T}$, $\mathrm{n}(\mathrm{N}, \mathrm{f}, \mathrm{T})$. Similarly, as [8], the real and imaginary parts of $\mathrm{n}$ have been obtained as analytical solutions, which lead, if a rectangular waveguide is considered, to the following definition for the optical gain,

$g(N, f, T)=2 k_{0} \operatorname{Im}\{n\}$

For a cylindrical waveguide with a radius $\mathrm{R}$, the optical gain is also a function of $\mathrm{R}$, that is, $\mathrm{g}(\mathrm{N}, \mathrm{f}, \mathrm{T}, \mathrm{R})$. Therefore, from the gain definition, besides the contribution of the losses in the material, given by $\operatorname{Im}\{n\}$, it should be also considered the losses associated with the curvature. The optical gain is then given by:

$g(N, f, T, R)=2 \frac{\operatorname{Im}\{v\}}{R}$

where $v=\gamma \mathrm{R}=(\alpha-\mathrm{j} \beta) \mathrm{R}$, with $\alpha$ and $\beta$, designated by damping constant and phase constant, respectively.

In rectangular waveguides, the confinement factor does not depend on R, and it is generally assumed, as a first approximation, independent of $\mathrm{n}$. In cylindrical geometries, $\Gamma$ is assumed dependent on $\mathrm{n}$ and, therefore, on $\mathrm{T}, \mathrm{f}$, and $\mathrm{R}$.

Summarizing, it is assumed that $\mathrm{n}(\mathrm{N}, \mathrm{f}, \mathrm{T}), \Gamma(\mathrm{n}, \mathrm{T}, \mathrm{f}, \mathrm{R})$ and $g(N, f, T, R)$, which means that the present model may be considered a generalization of the analysis presented in previous studies.

Under direct-current modulation, the current density, the photon density, and the carrier density are all time-dependent, according to:

$J=J_{0}+J_{M} \cos \omega t$

$S=S_{0}+\Delta S$

$N=N_{0}+\Delta N$

where $J_{0}$ is the bias current density and $N_{0}$ and $S_{0}$ are

respectively the steady-state carrier and photon densities.

Considering the linearization of the rate equations, (1) and (2) the power spectra for the signal $\left(S_{1}\right)$ and noise $\left(S_{2}\right)$ are obtained. Finally, the SNR is given by:

$S N R=\frac{P_{S}}{S_{2}(\omega=\Omega)}$

and $P_{S}=\int_{0}^{\infty} S_{1}(\omega) d \omega$

\section{RESULTS}

First, a rectangular waveguide is considered. This one corresponds to a double heterostructure semiconductor laser, and it is schematically represented in Fig. 1 b, with typical values for the laser and material parameters given in Table 1 of [7].

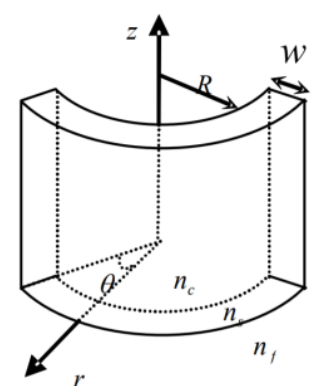

a)

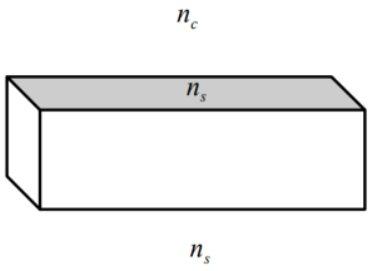

b)

Fig. 1. Schematic representation of the doubles heterostructure under analysis: a) bend structures and b) straight.

TABLE 1: VALUES OF THE PARAMETERS USED ON THE SIMULATIONS

\begin{tabular}{cccc}
\hline$n_{c}$ & $n_{s}$ & $n_{f}$ & $\mathrm{~W}$ \\
\hline 3.1 & 3.2 & 1 & $5 \mu \mathrm{m}$ \\
\hline
\end{tabular}

The modal gain is given by:

$g(N, f, T)=2 k_{0} n$

Contrarily to Wang, in this work the refractive index is considered as a function of the carrier concentration and frequency. The results obtained for SNR in the $(\mathrm{J}-\mathrm{f})$ plane are represented in Fig. 2, for $\mathrm{T}=-10{ }^{\circ} \mathrm{C}, \mathrm{T}=0{ }^{\circ} \mathrm{C}$ and $\mathrm{T}=$ $27^{\circ} \mathrm{C}$.

It is apparent from those curves that the SNR increases with frequency when $\mathrm{T}$ is made constant. However, it should be noticed that the SNR decreases with the temperature increase.
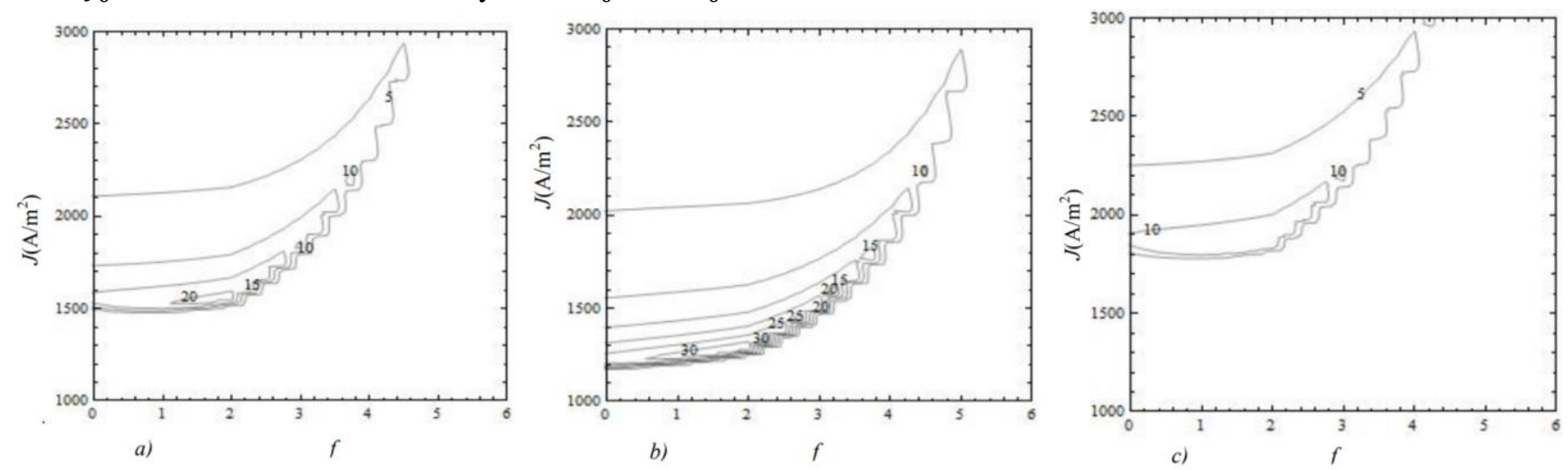

Fig. 2. The SNR in the ( $\mathrm{J}-\mathrm{f})$ plane when: a) $\left.\mathrm{T}=-10^{\circ} \mathrm{C}, \mathrm{b}\right) \mathrm{T}=0{ }^{\circ} \mathrm{C}$ and c) $\mathrm{T}=27^{\circ} \mathrm{C}$. 
For cylindrical waveguides (Fig 1a), the optical gain depends on R. Notice, that the results should lead to those previously reported for rectangular waveguides when $R$ approaches infinity. Fig. 3 shows the influence of the current density variation on the SNR when $\mathrm{R}=10 \mu \mathrm{m}$, for different values of the normalized frequency.

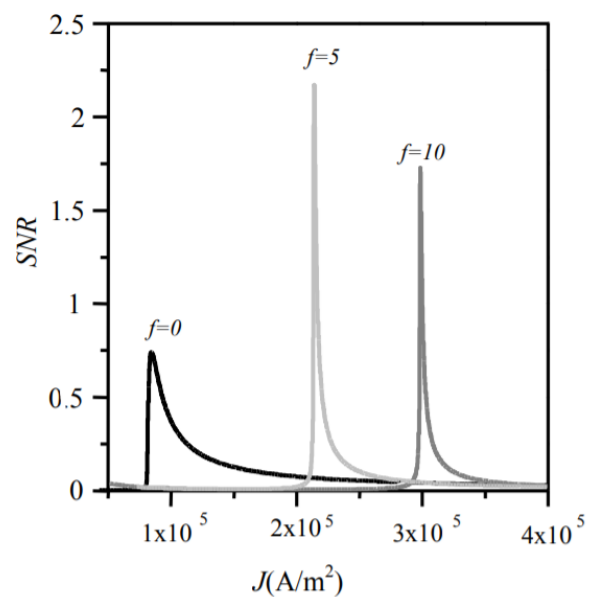

Fig. 3. SNR vs current density $\mathrm{J}$ for different normalized frequencies and $\mathrm{R}=10 \mu \mathrm{m}$.

As easily observed, SNR(J) does not have a monotonically evolution with the normalized frequency. Therefore, in order to ensure similar performances, the device needs less current density for smaller frequencies. Fig. 4 represents the influence of frequency on the current density associated to the maximum value of SNR, $J_{\max }(\mathrm{f})$, for several curvatures of the cylindrical waveguide, enhancing the fact that higher current densities are required for lower curvatures. This is directly related to the curvature losses, which decrease with the curvature radius.

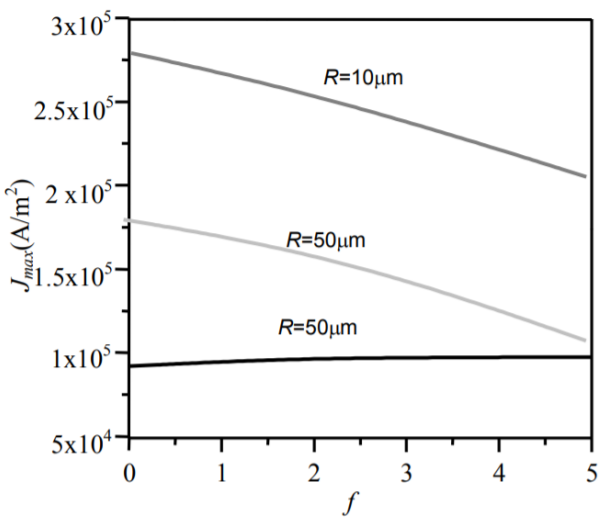

Fig. 4. $J_{\max }(\mathrm{f})$ for cylindrical waveguides with different radius.

\section{CONCLUSION}

The present model is a generalization of the analysis presented in previous studies by considering the influence of the curvature radius in non-rectangular waveguides on the relationship between the signal to noise ratio, the current density, and the frequency, according to the absorption model [10], in order to optimize the laser structure performance. These results may be special important when we are interested in coupling two structures, from the energetic point of view.

\section{ACKNOWLEDGMENT}

This work was supported in part by FCT/MCTES through national funds and in part by cofounded EU funds under Project UIDB/EEA/50008/2020.

\section{REFERENCES}

[1] R. Benzi, A. Sutera, A. Vulpiani, J. Phys. A 14 (1981) L453

[2] C. Nicolis, G. Nicolis, Tellus 33 (1981) 225

[3] S. Fauve, F. Heslot, Phys. Lett. A 97 (1983) 5.

[4] B. McNamara, K. Wiesenfeld, R. Roy, Phys. Rev. Lett. 60 (1988) 2626.

[5] G. Hu, T. Ditzinger, C.Z. Ning, H. Hanken, Phys. Rev. Lett. 71 (1993) 807.

[6] V. Berdichevsky, M. Gitterman, Europhys. Lett. 36 (1996) 161.

[7] J. Wang, Y.M.Bai, L. Cao, D.J. Wu, X. Y. Ma, Phys A 368 (2006) 31- 37.

[8] L. Bányai and S. Koch, Zeitschrift fur Physik B- Condensed Matter, 63 (1986).

[9] J. Torres, A. Baptista, V. M. Machado, EUROCON 2011 proceedings, Lisbon, 27-28 April 2011

[10] Joao Paulo N. Torres, V. Machado, and Antonio Baptista, A New Hybrid Finite Element Method: Electromagnetic Propagation in Bent Waveguides. IPhoJ 12.1 (2020): 2966256.

[11] João Torres, António Baptista, and Victor Maló Machado, Coupling analysis in concentric ring waveguides. Journal of lightwave technology 31.13 (2013): 2140-2145.

[12] R. A. Marques Lameirinhas, J. P. N. Torres and A. Baptista, The Influence of Structure Parameters on Nanoantennas' Optical Response. Chemosensors (2020), 8, 42.

[13] R. A. Marques Lameirinhas, J. P. N. Torres and A. Baptista, Sensors Based on Nanoantennas: Fundamentals. European Journal of Applied Physics (2020), 2, 3.

[14] João Torres, António Baptista, and Victor Maló Machado, Analysis of dielectric optical ring slab waveguides with a layered refractive index. 18th Telecommunications forum TELFOR 2010, Serbia, Belgrade (2010).

[15] J. Torres, A. Baptista and V. M. Machado, Analysis of dielectric optical ring slab waveguides with interband absorption. 2011 IEEE EUROCON - International Conference on Computer as a Tool, Lisbon (2011).

[16] R. A. Marques Lameirinhas, J. P. N. Torres and A. Baptista, A Sensor Based on Nanoantennas. Applied Sciences 10.19 (2020): 6837.

[17] T. Bonnal, et al, How to determine the complex refractive index from infrared reflectance spectroscopy?. SN Applied Sciences 2.12 (2020): $1-9$. 